\title{
Sistema de Gestión de Calidad en el Sector público. Una revisión literaria
}

\section{Quality Management System in the Public Sector. A literary review}

INFORMACIÓN DEL ARTÍCULO

Fecha de recepción: 11 de Septiembre de 2019.

Fecha de aceptación: 2 de Diciembre de 2019.

${ }^{I}$ Máster en Dirección, Universidad del Rosario - Colombia.

E-mail: juan.cepeda@urosario.edu.co Código ORCID:

https://orcid.org/0000-0002-6008-6569

${ }^{2}$ Estudiante Doctorado en Ciencias de la Dirección, Universidad del Rosario. Investigador de la Universidad del Rosario - Colombia.

E-mail: wilson.cifuentes@urosario.edu.co Código ORCID

https://orcid.org/0000-0001-5410-9763

CITACIÓN: Cepeda Duarte, J. P., \& Cifuentes Martínez, W. E. (2019). Sistema de Gestión de Calidad en el Sector público. Una revisión literaria. PODIUM, (36), 35-54

https://doi.org/10.31095/podium.2019.36.3

ENLACE DOI:

http://dx.doi.org/10.31095/podium.201 9.36 .3

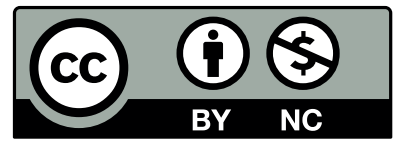

Juan Pablo Cepeda Duarte ${ }^{1}$, Wilson Eduardo Cifuentes Martínez ${ }^{2}$

\section{Resumen}

El sector privado ha influido en el sector público con técnicas para satisfacer las necesidades de los stakeholders, siendo una de estas el Sistema de Gestión de Calidad. Con la finalidad de analizar cómo ha mejorado la operación del sector público con ayuda de los Sistemas de Gestión de la Calidad, se realizó una revisión literaria de artículos en las bases de datos de Web of Science, Scopus y Proquest, y el repositorio normativo del Departamento Administrativo de la Función Pública en Colombia, para identificar su estado del arte en los últimos 10 años. Los hallazgos sugieren que para satisfacer las necesidades de las partes de interés de las organizaciones públicas se pueden utilizar esquemas de gestión como los de la calidad, facilitando la operación de las organizaciones pertenecientes al sector público.

\section{Palabras Clave:}

Administración Pública, cultura, gestión de la calidad, grupos de valor, sector privado, sector público, sistema de gestión.

\section{Clasificación JEL: M-M11.}

\begin{abstract}
The private sector has influenced the public sector with techniques to meet the stakeholders needs, one of them has been the Quality Management System. In order to analyze how the operation of the public sector has improved with the help of Quality Management Systems, a literary review of articles in the Web of Science, Scopus and Proquest databases, and the regulatory repository of the Colombian Public Function Administrative Department was elaborated to identify the state of the art in the last 10 years. The findings suggest that management schemes such as quality can be used to meet the stakeholders needs of public organizations, facilitating the public sector organizations operation.

\section{Keywords:}

Public Administration, Culture, Quality Management, Value Groups, Private Sector, Public Sector, Management System.
\end{abstract}

JEL Classification: M-M11. 


\section{Introducción}

Los gobiernos se enfrentan a la desconfianza actual de la sociedad por la mala calidad de los servicios prestados y la problemática para hacer frente a nuevos desafios, situación que dificulta la recuperación de la reputación perdida obligando a enfocar sus esfuerzos en prestar servicios que aporten significativamente a la vida de los ciudadanos (Aguilar, 2006). Esto pone de manifiesto la misionalidad del sector público, la cual consiste en elevar el bienestar de los ciudadanos en un ambiente de libertad y democracia promoviendo los servicios esenciales que el sector privado no presta o lo hace de manera insuficiente porque no hay demanda individual explicita (Valle, 2013).

Es por esto que la administración pública debe asumir nuevos retos, modernizando su gestión, apuntando a la necesidad de pensar en una administración moderna del siglo XXI y preocuparse por ser más eficiente $\mathrm{y}$ racional en el uso de los recursos (García, 2007). Estos cambios involucran la creación de esquemas de gestión en las organizaciones en el mundo caracterizados por afrontar cambios permanentes en el entorno y en la organización (de Souza y Macêdo, 2006; Felcman, Blutman, y Parnes, 2002; Gibson et al, 2006; Gutiérrez Pulido y Salazar, 2017; Hood, 1991; Hood, y Peters, 2004; Kaplan y Norton, 2002; Serna, 2008). Estos cambios han sido influenciados por el sector privado y la posibilidad de utilizar, desde este sector, nuevas técnicas que contribuyan a mejorar la gestión a través de buenas prácticas de calidad para ser adoptadas en lo público (Moyado, 2002). Estas prácticas administrativas hacen posible que la gestión pública se oriente a la creación de valor y satisfacción del ciudadano, ya sea como beneficiario de un servicio $\mathrm{y} / \mathrm{o}$ programa o como participante en el proceso formulación, ejecución y control de las políticas públicas. Algunos estudios sugieren que prácticas relacionadas con los Sistemas Integrados de Gestión mejoran la competitividad, perdurabilidad y éxito sostenible de las organizaciones (Rebelo, Santos y Silva, 2015).

La mejora de la gestión es un reto para la dirección y gerencia pública, de tal forma que se incremente la capacidad del Estado, para satisfacer las necesidades de la ciudadanía y los grupos de valor, a través del fortalecimiento del Modelo Integrado de Planeación y Gestión, donde un componente es el Sistema de Gestión de Calidad (SGC), y así generar confianza en las entidades públicas, aumentando la gobernabilidad y legitimidad del aparato público (DAFP, 2017). Para cumplir con este reto, se deben definir estándares de calidad para medir y hacer seguimiento a las características de los servicios prestados, con el fin de determinar desviaciones y definir acciones para mejorar la gestión (Elkomy y Cookson, 2018).

Otra práctica administrativa se refiere a la calidad del sector público, definida como la característica que le permite alcanzar el objetivo de equidad, que hoy es la meta más importante del estado (Tanzi, 2000). Por esto, el SGC es una 
herramienta estratégica del sector público que ayuda al cumplimiento de las metas del Estado, corrigiendo desviaciones en los procesos, disminuyendo la duplicidad de funciones, implementando acciones correctivas si fuese del caso, para facilitar el control político y ciudadano y ajustar los procedimientos a los requisitos exigidos en las normas internacionales para la creación de valor y satisfacción de sus usuarios (Congreso de la República de Colombia, 2003).

El esfuerzo de la administración pública no ha quedado sólo en implementar un SGC, sino la consolidación de un Sistema Integrado de Gestión, que apunte a un triple resultado final, incorporando temáticas enfocadas a la calidad (Norma ISO 9001), al medio ambiente (Norma ISO 14001), y a la salud y seguridad en el trabajo (Norma OHSAS 18001), que contribuyan a la sostenibilidad organizacional (Zeng, Shi $\&$ Lou, 2007). Por lo tanto, invertir en un Sistema Integrado de Gestión, aumenta el rendimiento económico de las empresas, aporta en materia ambiental y contribuye a satisfacer las necesidades de calidad de los usuarios (de Nadae, Carvalho y Rodrigues, 2019).

Por otro lado, entre los modelos del sector privado se destaca la ISO 9000 como desarrollo normativo, inicialmente formulada en 1987 desde la International Organization for Standardization (ISO), y que es revisada periódicamente para garantizar su actualización a las necesidades de las organizaciones y que se ajuste a la evolución del concepto de gestión de la calidad (Santos y Álvarez,
2007). Al respecto, la organización ISO es una red mundial que identifica cuáles normas internacionales son requeridas por el comercio, los gobiernos y la sociedad; y posteriormente las desarrolla junto con los sectores que las van a utilizar (Normas ISO 9001:2006; ISO 9001:2009; y ISO 9001:2015).

Para Callejas (2019), en Latinoamérica la norma que predomina es la ISO 9000, con el propósito fundamental de certificar los procesos de producción y sus servicios, además de servir de referencia como modelo para otras organizaciones. Adicionalmente, estas normas son base para los Sistemas de Gestión de Calidad en lo público y se obtienen gracias a que la ISO agrupa organizaciones para motivar alrededor del mundo la generación de normas para la producción de productos y/o la prestación de servicios en diferentes tipos de organizaciones (Lizarzaburo, 2016). Las normas descritas definen a la gestión de la calidad con fundamento en la orientación al cliente independiente del tipo de organización que ejecute las actividades, pero basado en un sistema que permita mantener y mejorar la operación de esta (Fernández, 2010; Joiner, 1995).

Para profundizar en lo expuesto, se ejecuta una revisión literaria de artículos científicos en un periodo de tiempo desde el 2009 al 2019, con el propósito de identificar la evolución y el estado del arte y de esta manera responder a la pregunta de investigación ¿Cuáles son las iniciativas del sector público utilizadas para mejorar su gestión a partir de la implementación de un Sistema de Gestión de Calidad? 


\section{Revisión de literatura}

La metodología de revisión seguida en este artículo permite identificar qué se conoce del tema en cuestión, qué se ha investigado y qué aspectos permanecen desconocidos, en la cual el revisor recoge datos, los analiza y extrae conclusiones (Guirao-Goris, Olmedo Salas y Ferrer Ferrandis, 2008). Para el efecto, se realizó una revisión sistemática de literatura que permitió lograr una síntesis para responder al tema de investigación propuesto (Francesc, Duch Gavald \& Gisbert Cervera, 2014).

Específicamente, se hizo una revisión de la literatura del desarrollo de los Sistemas de Gestión de Calidad en el sector público, con el objetivo de identificar los principales avances, los puntos en común que comparten los autores, y la información relevante recopilada para responder a un vacío de conocimiento en este tema relacionado con la aplicación de estos sistemas, base para la gestión pública (Calle, 2016). Para esto, se incluyeron tres bases de datos: Web of Science, Scopus y Proquest, y el repositorio de normatividad del Departamento Administrativo de la Función Pública - DAFP de Colombia.

Se analizó a Colombia porque es el segundo país con mayor número de certificaciones de Sistemas de Gestión de la Calidad obtenidas en los últimos diez años en Latinoamérica, pero el primero en porcentaje de crecimiento de estas en el mismo periodo (ISO, 2019).

El artículo se soporta en la teoría clásica articulada por Max Weber y Frederick
Taylor que consideran que las organizaciones públicas se representan por la racionalidad cuando sus objetivos son específicos y formalizados (Osbome, 2006). Así, la teoría de la organización argumenta que la organización pública se caracteriza por la multiplicidad de propósitos, funciones y sistemas que crean un sistema complejo que lucha para optimizar el valor social agregado, reconociendo además la relevancia de la estrategia de gestión del desempeño como marco de establecimiento de objetivos - gestión por objetivos (Osborne, 2006).

En el mismo sentido, el estado del arte para la gestión de la calidad arroja que el autor seminal es Deming (1982), quien plantea a la calidad como base de la gestión y mejora continua en las organizaciones; de allí que Deming sea considerado padre de la tercera revolución industrial (Khadra, Barqawi \& Alramahi, 2012; Lasrado, 2017; Octavian, Stãnciuc y Viorel, 2013; Phusavat et al, 2009). Con respecto al término Sistemas de Gestión de la Calidad en el sector público a nivel global, se puede afirmar que ha sido poco estudiado y en Colombia es relativamente nuevo (a partir de 2003), con la obligatoriedad de implementar un SGC en la Rama Ejecutiva del Poder Público (Congreso de la República de Colombia, 2003). Esto se complementa con el hecho de que la gestión pública no ha sido ajena a las tendencias de mejoramiento en la gestión que en algunos casos ha tenido su origen en el sector privado (Camarasa, 2004). Esto ha permitido que en las dos últimas décadas se tenga mayor confianza y transparencia en la administración pública a través de la generación de bienes y servicios que 
Tabla 1 .

Evolución de la ISO 9001

\begin{tabular}{cl}
\hline Año & \multicolumn{1}{c}{ Características } \\
\hline 1987 & $\begin{array}{l}\text { Publicada por primera vez en 1987. Ha sido utilizada alrededor del mundo para mejorar la } \\
\text { calidad de productos y servicios, y optimizar la gestión y eficiencia. }\end{array}$ \\
1994 Cambios muy prescriptivos, se centraron mucho en empresas fabriles. \\
2000 Pasó a ser una norma de gestión de a calidad y no de control de calidad. Se introdujo el Enfoque \\
de procesos.
\end{tabular}

Fuente: El autor a partir de Alzate (2017).

responden a las necesidades de sus grupos de valor.

Para comprender la importancia que sobre los Sistemas de Gestión de la Calidad del sector público ha tenido el sector privado, en la Tabla 1 se presentan las principales características de la evolución de la Norma ISO 9001, que especifica los requisitos para un SGC en una organización.

\section{Metodología}

Los esfuerzos del sector público para mejorar su gestión han incluido como herramienta la implementación y mejora de los Sistemas Integrados de Gestión y los Sistemas de Gestión de Calidad para reducir los costos para los ciudadanos en la interacción con el sector público y brindar servicios de calidad (Barros y Rodríguez, 2004). Para revisar estos esfuerzos se ejecutó una revisión literaria, y se estableció su origen y desarrollo en respuesta a la pregunta de investigación. De manera complementaria se realizó el análisis para Colombia a través de una revisión de la normatividad relacionada con el tema de los Sistemas de Gestión de Calidad, debido al amplio desarrollo de esta materia en este país. Para lo anterior, se tienen en cuenta los siguientes pasos (Beltrán, 2005; Ramírez, Meneses, y Florez, 2016): 1) Identificación, 2) Inclusión y Exclusión, 3) Plan de búsqueda, 4) Resultados y discusión, y 5) Conclusiones y recomendaciones.

\section{Identificación}

En esta etapa se hace referencia a las fuentes de información revisadas, los repositorios utilizados para la selección de los ítems (palabra clave, conceptos) que van a servir de soporte para el trabajo desarrollado.

Para la revisión literaria se utilizaron las bases de datos de Web of Science, Scopus y Proquest, siendo estos algunos de los repositorios más importantes de publicación científica para realizar consultas de investigación. Asimismo, fueron seleccionados teniendo en cuenta la facilidad para acceder a la documentación, y el número y tipo de 
documentos disponibles.

Web of Science es una de las bases de datos más utilizadas por la comunidad científica, y para complementarla surgió Scopus, siendo estas bases de datos las herramientas que han permitido masificar y evaluar la información científica (Legaz et al, 2016). Asimismo, Proquest es una base de datos que se dedica a proporcionar información y servicios de biblioteca a los investigadores, y además incluye fuentes autorizadas, páginas digitales y la mayor colección de tesis doctorales, entre otros (Clement y Rascoe, 2013).

De igual forma, se consultó el repositorio de información normativa del DAFP, teniendo en cuenta que en este sitio se encuentran consolidadas las normas y conceptos asociados a la Tabla 2.

Criterios de Elegibilidad

\begin{tabular}{cc}
\hline Descripción & Criterio \\
\hline
\end{tabular}

\section{Criterios de inclusión}

Palabras clave (inglés y español) Sector público

Public Sector

Sistema Integrado de Gestión Integrated Management System

Sistema de Gestión de la Calidad Quality Management System

Términos alternativos Posibles sinónimos

Ninguno

Ninguno

Criterios de exclusión

Campo del conocimiento

Tipo de exclusión administración pública colombiana (Congreso de la República de Colombia, 1992). Es importante revisar la normatividad asociada al sector público, teniendo en cuenta que Colombia es un Estado de derecho organizado de forma de República unitaria, descentralizada, democrática, participativa y pluralista (Asamblea Nacional Constituyente, 1991). De igual manera, la normatividad es un referente para el estudio, teniendo en cuenta que tiene el carácter general de mandar, permitir y castigar tanto en lo público como en lo privado (Congreso de los Estados Unidos de Colombia, 1873).

\section{Inclusión y Exclusión}

Los criterios que sustentan la elegibilidad y los aspectos que reducen los errores de identificación y sesgo se resumen a continuación (Tabla 2 ). 
Tabla 3

Resumen Búsqueda Inicial

\begin{tabular}{lrrr}
\hline \multicolumn{1}{c}{ Palabras clave } & $\begin{array}{c}\text { Web of } \\
\text { Science }\end{array}$ & Scopus & Proquest \\
\hline Public Sector & 40130 & 105697 & 47384 \\
Integrated Management System & 39316 & 111630 & 14879 \\
Quality management System & 43580 & 202046 & 104684 \\
Subtotal & 123026 & 419373 & 166947 \\
Sector público & 20 & 521 & 35 \\
Sistema Integrado de Gestión & 0 & 5 & 0 \\
Sistema Gestión de la Calidad & 0 & 49 & 0 \\
Subtotal & 20 & 575 & 35 \\
\hline Total & $\mathbf{1 2 3 0 4 6}$ & $\mathbf{4 1 9 9 4 8}$ & $\mathbf{1 6 6 9 8 2}$ \\
\hline
\end{tabular}

Fuente: Información tomada de base de búsqueda Web of Science, Scopus y Proquest (2019).

\section{Plan de Búsqueda}

Se ejecuta la búsqueda en Web of Science, Scopus y Proquest, se revisan los resultados que apuntan a resolver la pregunta descrita en el apartado de metodología y no se tienen en cuenta los que hacen referencia a sectores diferentes al público. De igual forma, se eliminan los artículos repetidos y se tienen en cuenta los resultados arrojados en los años 2009 al 2019 para precisar el estado del arte a partir de las palabras clave que soportan el alcance de la investigación (Tabla 3).

Tendencia de la búsqueda en cada base de datos

En la Figura 1, se observa que la producción de publicaciones en la base de datos Web of Science, tuvo una ligera tendencia al aumento a partir del $2009 \mathrm{y}$ hasta el 2018. De igual forma, aparecen tres líneas de tendencia referente a los resultados en inglés, con respecto a los

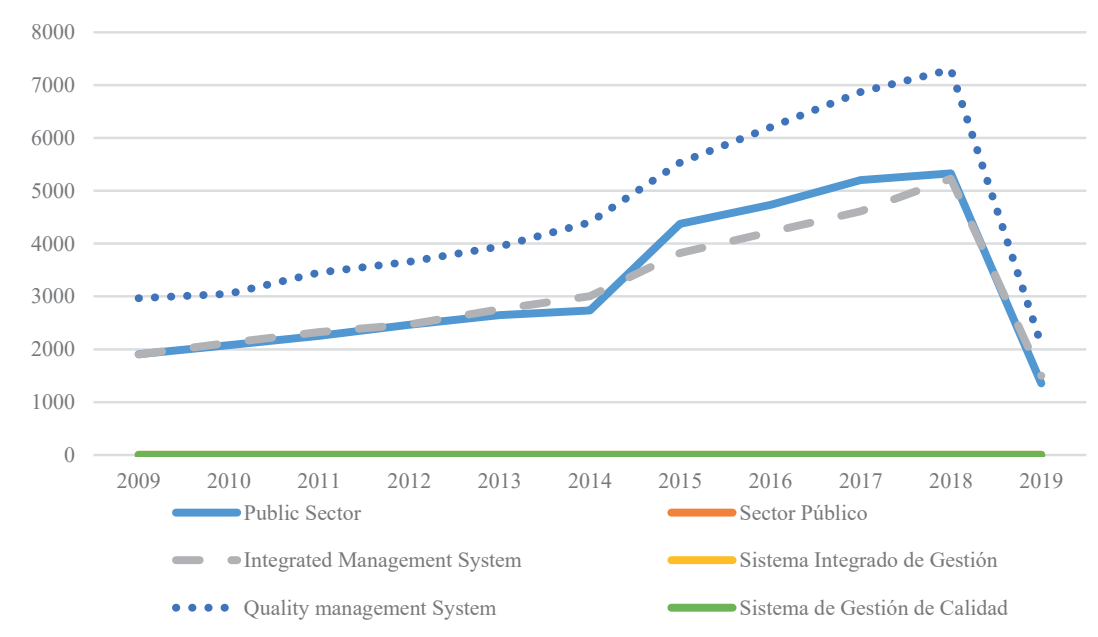

Figura 1. Tendencia palabras claves - Base de datos Web of Science.

Fuente: Base de datos Web of Science. 


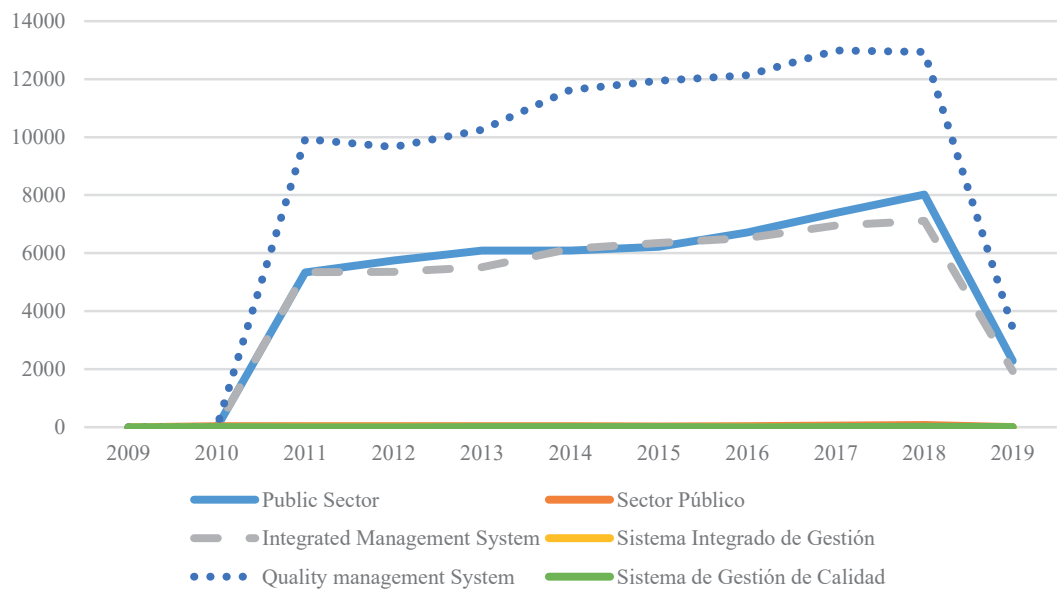

Figura 2. Tendencia palabras claves Base de Datos Scopus. Fuente: Base de datos Scopus.

resultados en español no se muestra la tendencia. Con relación a las palabras clave: Sistema Integrado de Gestión y Sistema de Gestión de Calidad, el resultado fue cero.

Para la tendencia en Scopus, la Figura 2 refleja una ligera tendencia al aumento a partir del 2009 y hasta el 2018. En el mismo sentido, aparecen sólo tres líneas de tendencia visibles de los resultados en inglés, en español aparece sobre la línea horizontal la tendencia del sector público.
Para la base de datos Proquest, en la Figura 3 se observa que las publicaciones con el término Public Sector tuvieron una ligera disminución en el 2013 y 2014, y en 2015 hay un aumento, ahora bien, la palabra Integrated Management System, tuvo un repunte en 2015 y Quality Management System se mantiene constante en los años de análisis.

Es importante mencionar que el análisis de los resultados sólo se hace con los años 2009 a 2015, porque al realizar el

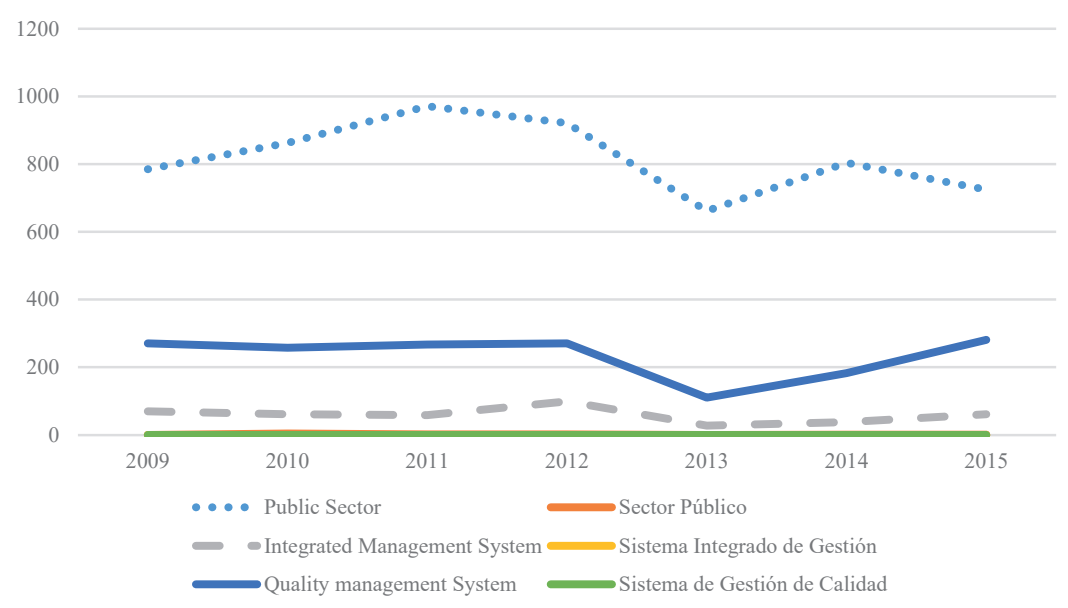

Figura 3. Tendencia palabras claves - Base de Datos Proquest.

Fuente: Base de datos Proquest. 


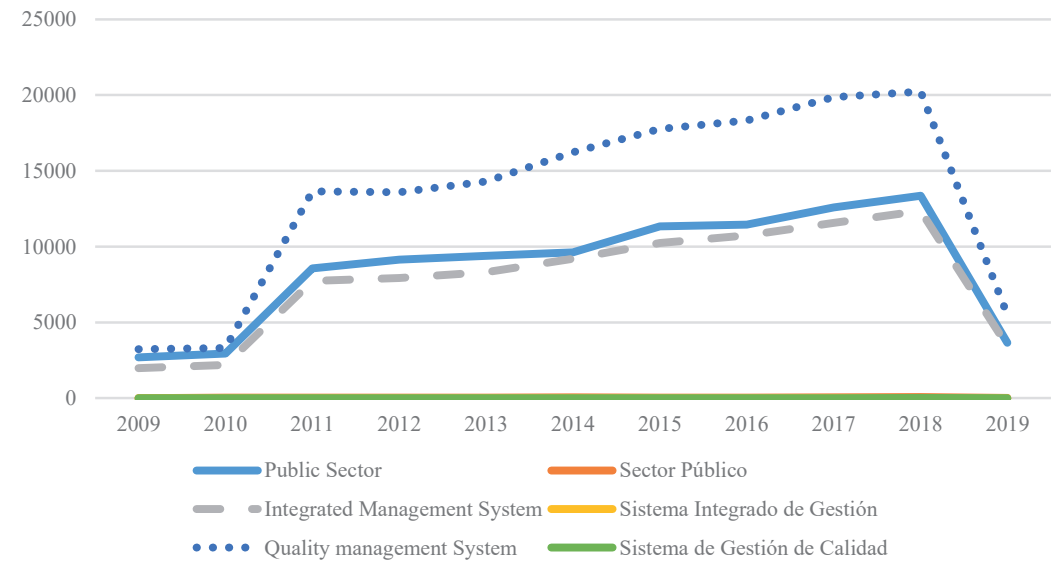

Figura 4. Tendencia Base de Datos Consolidada.

Fuente: Base de datos Web of Science, Scopus, Proquest.

filtro en la base de Datos Proquest, no arrojó resultados de los años 2016 al 2019.

\section{Tendencia Base de Datos Consolidada}

La tendencia de producción de las palabras de manera consolidada en las tres bases de datos tuvo un leve incremento en la producción en los últimos años (Figura 4).

La mayor producción en inglés se concentra en Estados Unidos con el 22\%, seguido de Inglaterra con un $7 \%$ y en tercer lugar la República China con el $5 \%$. La producción en español se concentra en Brasil con 33\%, seguido de España con $20 \%$ y en tercer lugar Chile con $7 \%$.

\section{Ecuaciones Booleanas}

En este punto resulta necesario incorporar el uso de operadores booleanos para generar mejores resultados a partir de las búsquedas ejecutadas, teniendo en cuenta la gran cantidad de información obtenida sobre la temática de investigación (Villegas, 2003). A través de este proceso se reducen los más de 700.000 documentos incluidos en la búsqueda inicial.

Para el ejercicio realizado, al momento de efectuar consultas a Web of Science, Scopus y Proquest, se utilizó el operador AND, de la siguiente manera:

- ((“"Integrated Management System") AND ("Public Sector") AND (“Quality management System")),

- ("Public Sector") AND ("Quality management System"),

- (("Sistema Integrado de Gestión”) AND ("Sector público") AND ("Sistema de Gestión de la Calidad")),

- ("Sector público") AND ("Sistema de Gestión de la Calidad")

Las búsquedas se realizaron en inglés y español; en las tres bases el idioma predominante es el inglés con 44 documentos (Figura 5). En la Tabla 4 se resumen los artículos obtenidos. 


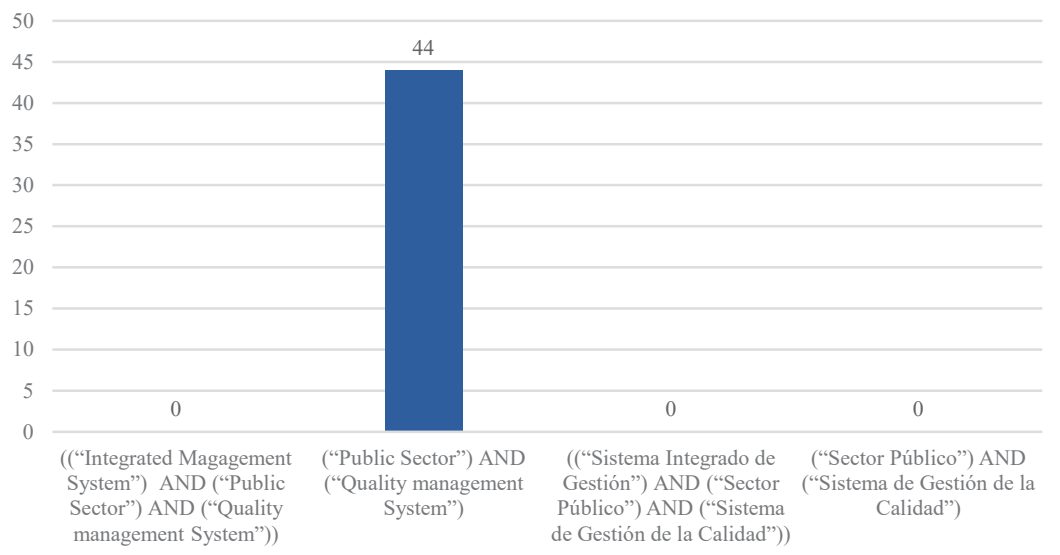

Figura 5. Total de Resultados Ecuaciones Booleanas - inglés y español.

Fuente: Bases de Datos Web of Science, Scopus, Proquest.

\section{Web of Science}

De los 10 resultados arrojados en la búsqueda en esta base de datos, tres son de años anteriores al 2009, los cuales no entran en el rango de años definidos. De los cuatro que quedan, se tienen en cuenta dos asociados a la administración y la Ingeniería Industrial y de estos uno tiene acceso abierto y el otro no, además este artículo esta repetido en la base de datos Scopus. Por lo anterior, para esta base de datos solo queda un artículo para el siguiente paso.

\section{Scopus}

De los 33 resultados arrojados en la búsqueda en esta base de datos, trece son de años anteriores al 2009, los cuales no entran en el rango de años definidos. De los veinte que quedan se tienen en cuenta diez asociados a la administración que están en el rango de tiempo. De estos, ocho tienen acceso abierto y dos no, lo que permite obtener ocho documentos para el siguiente paso.

\section{Proquest}

Del artículo arrojado en la búsqueda, este se descarta porque es de un año anterior al 2009. De acuerdo con lo anterior, el análisis de las tres bases de datos nos arroja un resultado de 10 artículos que se ajustan a los criterios de

Tabla 4.

Resumen Ecuación Booleana (“Public Sector”) AND (“Quality Management System”)

\begin{tabular}{lccl}
\hline \multicolumn{1}{c}{ Base } & $\begin{array}{c}\text { No de } \\
\text { documentos }\end{array}$ & $\mathbf{\%}$ & \multicolumn{1}{c}{ Países } \\
\hline Web of Science & 10 & 22,72 & Tailandia (2), Australia (1) \\
Scopus & 33 & 75 & Finlandia (1), Otros (6) \\
Proquest & 1 & 2,27 & Finlandia (3), Grecia (3) \\
Total & 44 & 100 & Emiratos Árabes Unidos (3) \\
\hline
\end{tabular}

Fuente: Bases de datos Web of Science, Scopus, Proquest (2019). 
Tabla 5 .

Resumen artículos seleccionados para revisión final

\begin{tabular}{lcc}
\hline \multicolumn{1}{c}{ Nombre del Artículo } & Autor & $\begin{array}{c}\text { Año de } \\
\text { Publicación }\end{array}$ \\
\hline $\begin{array}{l}\text { A four-fold regional-specific approach to TQM: The } \\
\text { case of South Eastern Europe }\end{array}$ & Psychogios & 2010 \\
$\begin{array}{l}\text { Governance using ISO 9001:2000 challenges and } \\
\text { barriers: empirical study applied on the Jordanian } \\
\text { private mobile companies }\end{array}$ & Khadra, Barqawi \& Alramahi & 2012 \\
$\begin{array}{l}\text { Implementing TQM initiatives in public service } \\
\text { organisations: Case of academic libraries }\end{array}$ & Tuomi, Ajmal \& Helo & 2013 \\
$\begin{array}{l}\text { Integrarea Managementului Total al Calitãpii în } \\
\text { administrapia publicã din România }\end{array}$ & Octavian, \\
$\begin{array}{l}\text { Performance measurement: roles and challenges } \\
\text { The excellence quest: a study of business excellence } \\
\begin{array}{l}\text { award-winning organizations in UAE } \\
\text { TQM Practices in Public Sector: }\end{array}\end{array}$ & 2013 \\
$\begin{array}{l}\text { Quality management system as the basiss of quality } \\
\text { assurance at všb-technical university of ostrava. }\end{array}$ & Phusavat, Anussornnitisarn, & 2009 \\
$\begin{array}{l}\text { The Impact off Quality Management Implementation } \\
\text { within the Romanian Local Public Institutions }\end{array}$ & Lasrado & 2017 \\
\hline
\end{tabular}

Fuente: Bases de datos Web of Science, Scopus (2019).

inclusión y exclusión, pero uno de estos no tiene acceso abierto, por tal motivo se descarta y en total quedan nueve.

Adicional a lo anterior se revisaron los 9 artículos con el fin de establecer si el contenido efectivamente apunta a la pregunta de investigación, dando como resultado que todos lo hacen. Es importante mencionar que uno está enfocado en el Sector privado, pero al ser leído a profundidad, también contribuye al desarrollo del tema. La descripción se presenta en la Tabla 5.

\section{Resultados}

Una vez revisados los nueve artículos que arrojó la ecuación booleana ("Public Sector”) AND ("Quality Management
System") se describen los principales aportes que contribuyen al entendimiento del desarrollo de la temática en el sector público, pero antes, es importante mencionar que el $100 \%$ están escritos en inglés, y que una vez revisado el contenido, algunos autores comparten que el autor dominante en el tema de la calidad es William Edwards Deming considerado el padre de la tercera Revolución Industrial (Fernández, 2010). De igual manera, en el sector público el término es relativamente nuevo y está influenciado por la globalización.

Se identificó la importancia de ofrecer productos y servicios de calidad a sus clientes independientemente de si el sector es público o privado, con el fin de 
contribuir a la supervivencia de las organizaciones (Octavian et al, 2013). Si bien el término de calidad inició en el sector privado y las primeras iniciativas surgieron en el sector manufacturero, la temática ha ganado relevancia en la administración pública (Tuomi, Ajmal, y Helo, 2013). De igual forma, se identificó la importancia estratégica que tiene el SGC para la alta gerencia, teniendo en cuenta que muestra una visión clara del negocio, permitiendo monitorear y hacer seguimiento a todos los procesos de la organización (Lasrado, 2017; Psychogios, 2010; Tuomi et al, 2013).

Actualmente, tanto las organizaciones públicas como privadas se ven sometidas a una presión constante de los mercados globales, que generan mayor competencia y necesidades de los clientes que dificultan la supervivencia de las organizaciones, por tal motivo estas deben centrarse en la calidad y clientes (Octavian, Stanciuc, y Viorel, 2013). En concordancia con lo expuesto anteriormente, las organizaciones privadas y públicas trabajan en entornos complejos que disparan la necesidad de contar con estructuras de gestión $\mathrm{y}$ medición orientadas a la calidad en las actividades que se realizan y que incluyen avances tecnológicos que generan cambios continuos en las áreas de servicios y generan constantemente nuevas expectativas por parte de la sociedad, lo que crea la necesidad de implementar Sistemas de Gestión de la Calidad (Androniceanu, 2012).

De otro lado, el enfoque inicial que se le dio a la Gestión de la calidad estaba relacionado con la inspección como filosofía. El concepto ha evolucionado, incorporando el bienestar, la sociedad, el medio ambiente y la sostenibilidad hacia una gerencia estratégica y el mejoramiento de la gestión como parte de la supervivencia (Ajmal et al, 2016). De manera complementaria, independientemente de si es el sector público o privado, las organizaciones se ven presionadas por el entorno y los mercados globales para implementar prácticas que apunten a satisfacer necesidades de sus clientes (Santiso, 2016).

La evolución descrita refleja que la administración pública está implementando bienes y servicios de mayor calidad, que apuntan a satisfacer la necesidad de los grupos de valor, pero el concepto de cliente es diferente al del sector privado, porque los clientes tienen diferentes demandas, necesidades $\mathrm{y}$ expectativas con respecto a la administración pública (Psychogios, 2010). Así, para cumplir con la misionalidad de las organizaciones públicas, estas han utilizado el SGC como una herramienta genérica aplicable a cualquier organización, pero se sugiere tener en cuenta el entorno donde se va a desarrollar (Ajmal et al, 2016). De igual manera, es necesario tener en cuenta que indistintamente del tipo de organización, se debe revisar los beneficios que la implementación de sistemas de gestión trae a los grupos de valor a partir de la provisión de bienes y servicios de calidad en el marco de una política de calidad que incluya la satisfacción de necesidades y 
mejora como pilares (Ajmal et al, 2016; Androniceanu, 2012; Khadra et al, 2012).

Adicional a estos aspectos, es importante identificar las actividades desarrolladas y si estas apuntan a cumplir con las expectativas de los clientes, realizar la medición como herramienta de gestión y aprendizaje de mejoramiento continuo para la organización, y la necesidad de definir criterios de rendimiento para poder controlar los procesos de gestión (Hutyra, 2009; Phusavat et al, 2009). Por otro lado, los SGC en lo público se ven influenciados por la cultura organizacional, la cual depende del estilo de liderazgo gerencial, y este puede afectar el éxito de su implementación (Ajmal et al, 2016). En este punto coinciden los autores señalando que el compromiso, ayuda y liderazgo de la gerencia es indispensable, para movilizar a la organización en pro de un SGC (Lasrado, 2017; Psychogios, 2010; Tuomi et al, 2013).

En cuanto a los resultados de la búsqueda y análisis de la normatividad relacionada con la gestión en el sector público de Colombia, se realizó la búsqueda teniendo en cuenta la normatividad asociada a los Sistemas de Gestión de Calidad y Sistemas Integrados de Gestión que permiten observar la evolución del tema del mejoramiento de la gestión y los esfuerzos de la administración pública en Colombia por implementarlos. Como referente inicial, se tiene que, en Colombia en 1991, se modifica la Constitución de 1986 y se expide una nueva Constitución, con el fin de modernizar y ajustar a la realidad los derechos y deberes de los ciudadanos y estructurar y organizar el Estado. Además, se establece que la función administrativa está al servicio de los intereses generales y se desarrolla con fundamento en los principios de igualdad, moralidad, eficacia, economía, celeridad, imparcialidad y publicidad (Asamblea Nacional Constituyente, 1991). En 1992, el Gobierno Nacional de Colombia moderniza y reestructura el Departamento Administrativo del Servicio Civil, y cambia su denominación al DAFP, con el fin que esta entidad defina políticas administrativas de gestión pública y vele por los temas de recursos humanos al servicio del Estado (Congreso de la República de Colombia, 1958; Presidencia de la República, 1992). Por otro lado, en 1998 nuevamente en Colombia se generan esfuerzos por parte del Estado, con el propósito de organizar el funcionamiento de las entidades del orden nacional y definir entre otros temas el Sistema de Desarrollo Administrativo de las entidades públicas conformado por una serie de políticas, estrategias y mecanismos para mejorar el manejo de los recursos humanos, técnicos, materiales, físicos y financieros de las entidades de la Administración (Congreso de la República de Colombia, 1998).

En el 2003, surge otra iniciativa en la Administración Pública, con el propósito de estandarizar y mejorar el servicio a la ciudadanía, esta vez se crea el SGC de obligatoria implementación en la Rama Ejecutiva del Poder Público y en otras entidades prestadoras de servicios, el cual está enfocado en procesos y busca 
evaluar y hacer más eficiente la gestión en términos de calidad y satisfacción de los grupos de valor (Congreso de la República de Colombia, 2003).

De acuerdo con lo anterior y con el fin de tener una herramienta que establezca los requisitos para la implementación de un SGC, se adopta la Norma Técnica de Calidad en la Gestión Pública NTCGP 1000 versión 2004 y establece los requisitos mínimos para la implementación de un SGC en Colombia y las entidades obligadas (Presidencia de la República, 2004). Teniendo en cuenta que surgen nuevas expectativas $\mathrm{y}$ necesidades en el sector público, las normas emitidas que se relacionan con Sistemas de Gestión de la Calidad son reemplazadas por el Decreto 1080 del 2015.

En 2005, con el propósito de evaluar la estrategia y la gestión de las entidades del Estado colombiano, se implementa el Modelo Estándar de Control Interno MECI, en el cual se establecen procedimientos y estrategias para llevar a la realidad principios relacionados con estos temas (DAFP, 2005). Teniendo en cuenta que en Colombia desde el 2003 se tiene la experiencia de la implementación de un SGC en la Administración Pública y que en el 2009 se identifican otras necesidades y se quiere ir de la mano con las actualizaciones a la ISO 9000, fue necesario actualizar la NTCGP versión 2004 a la versión 2009 (Presidencia de la República, 2009). Otro esfuerzo que desarrolla la Administración Pública en Colombia es la integración de la planeación y la gestión, en el cual se adopta el Modelo Integrado de
Planeación y Gestión, que busca mejorar la Eficiencia Administrativa, a través de la prestación de un mejor el servicio a los grupos de valor (DAFP, 2012). La norma base de este modelo fue derogada por el Decreto 1083 de 2015, el cual actualiza el Modelo Integrado de Planeación y Gestión.

En 2014, se actualiza el MECI para coadyuvar a responder los nuevo retos y desafíos de la administración pública en Colombia (Presidencia de la República, 2014). Teniendo en cuenta que siguen los avances y la identificación de nuevas necesidades en la gestión pública, la actualización descrita es derogada por el Decreto 1083 del 2015 (Presidencia de la República, 2015). En el mismo año Colombia tiene un nuevo Plan Nacional de Desarrollo 2014 -2018 denominado "Todos por un nuevo país", el cual hace referencia a la integración en un solo Sistema de Gestión, el Sistema de Gestión de la Calidad y el de Desarrollo Administrativo, además del Sistema de Control Interno, para articular, unificar esfuerzos, estandarizar lenguaje y evitar desgastes administrativos y reprocesos (Congreso de la República de Colombia, 2015). Nuevamente en 2017, y para avanzar en la madurez del Modelo Integrado de Planeación y Gestión en la Administración Pública Colombiana, este modelo tiene una nueva actualización que busca hacer más eficiente la Administración pública (DAFP, 2017).

Como se puede evidenciar, la administración pública colombiana ha implementado una serie de iniciativas a través de la expedición de normas, con el 
fin de tener un sistema propio de calidad, el cual es influenciado por las normas ISO. El propósito fundamental de estas iniciativas es una gestión pública, más eficiente y transparente enfocada en cumplir las necesidades y expectativas de los grupos de valor. Por último, es importante señalar que la administración pública colombiana a partir del 2003, ha repensado su quehacer, enfocado en ser más trasparente $\mathrm{y}$ eficiente en la prestación de servicios, teniendo en cuenta las necesidades y expectativas de sus grupos de valor, implementando la norma NTCGP, la cual ha tenido una serie de actualizaciones, para mejorar continuamente y ajustarse a las nuevas realidades de la administración pública.

\section{Conclusiones}

La revisión efectuada permitió identificar que el sector público ha tenido que implementar estrategias de calidad para restablecer la confianza y reputación de sus entidades, respecto de los servicios ofrecidos. Al examinar el fundamento teórico y práctico de estas estrategias se puede identificar la influencia del sector privado reflejada en herramientas de medición y desempeño como la ISO 9000, que apunta a la satisfacción de los grupos de valor a través de Sistemas de Gestión de la Calidad. Estas herramientas se han tomado como alternativa para mejorar la gestión pública y como fuente de satisfacción de las necesidades de los grupos de valor del sector público.

Por otra parte, la revisión efectuada permitió reconocer que los sectores privado y público se enfrentan a un cambio permanente del entorno y las condiciones para satisfacer las necesidades cada vez más complejas de sus grupos de valor, que involucran además los aspectos propios de la naturaleza de cada organización que obliga a poner especial interés a los sistemas de gestión, especialmente en aquellas de naturaleza pública.

Otro resultado de la revisión literaria es que la mayoría de los autores analizados coinciden con la importancia del estilo y liderazgo de la alta gerencia en el éxito de implementación de los Sistemas de Gestión de Calidad. Igual situación se encuentra cuando se analiza el reconocimiento y entendimiento de la cultura del país y de la organización donde se va a implementar un SGC, como factor de éxito o de fracaso de la implementación de este tipo de herramientas para mejorar la gestión pública. Esta situación se complementa con la idea de que existen barreras para la implementación de los Sistemas de Gestión de Calidad en lo Público como son la falta de liderazgo y un bajo compromiso de la gerencia.

Con respecto a Colombia, el fenómeno de los Sistemas de Gestión de la Calidad en la administración pública es relativamente nuevo y se remonta al año 2003, con la expedición de normatividad que obliga a las entidades de la rama ejecutiva a su implementación. En sus inicios se planteó como un modelo estratégico de mejoramiento continuo y ello ha permitido optimizar la gestión y coadyuvar al cumplimiento de la misionalidad del Estado, a través de la 
prestación de bienes y servicios que satisfacen las necesidades de los ciudadanos.

La evolución en la aplicación en el sector público colombiano de los estándares de calidad ha generado la necesidad de enfocar esfuerzos adicionales para consolidar un Sistema Integrado de Gestión, apuntando a un tripe resultado final (calidad, ambiental y seguridad y seguridad del trabajo). Asimismo, esta evolución ha sido soportada con la expedición de normatividad relacionada con la actualización de los sistemas de gestión y mejoras que faciliten la puesta en marcha de estas iniciativas en las organizaciones del sector público. No obstante, se presentaron limitaciones para el desarrollo de la revisión de la literatura como el acceso restringido de los artículos y lo poco que se ha escrito respecto a lo público.

Se propone para futuras líneas de investigación que se indague sobre temas que pueden generar valor en el entendimiento de la implementación de un Sistema de Gestión de Calidad en lo público: ¿cuál es la motivación de las organizaciones públicas para la implementación de un Sistema de Gestión de Calidad?, ¿cuáles pueden ser las barreras en la implementación de los Sistemas de Gestión de Calidad en el sector público?, ¿de qué manera el liderazgo de los gerentes públicos influye en la implementación de Sistemas de Gestión de la Calidad?, ¿cómo incorporar el cambio del entorno a los sistemas de gestión de lo público?, ¿quiénes son los actores clave para la sostenibilidad de los sistemas de gestión?, ¿cuál es el futuro de los Sistemas de Gestión de la Calidad en lo público? Además, se podría realizar una investigación que apunte a identificar si los Sistemas de Gestión de Calidad contribuyen a que el Estado cumpla con su misionalidad en forma eficiente para afirmar que la gestión del sector público es de alta calidad (Tanzi, 2000). Estas iniciativas pueden ser apalancadas con la idea de que los Sistemas de Gestión de la Calidad en lo público han recibido la suficiente atención y actualización para afirmar que es una oportunidad para mejorar el servicio público.

\section{Referencias}

Aguilar, L. (2006). Gobernanza y gestión pública. México D.F.: Fondo de Cultura Económica.

Ajmal, M., Toumi, V., Helo, P., y Sandhu, M. (2016). TQM Practices in Public Sector: Case of Finnish Healthcare Organizations. International Journal of Information Systems in the Service Sector, 8(1), 34-44.

Alzate, A. (2017). ISO:2015 base para la sostenibilidad de las organizaciones en países emergentes. Revista Venezolana de Gerencia, 22(80), 33-49.

Androniceanu, A. (2012). The impact of quality management implementation within the Romanian local public institutions. Quality Management, 13(127), 96-99.

Asamblea Nacional Constituyente. (1991). Constitución Política de Colombia. Por el cual se expide la Constitución Política de Colombia. Publicada en El Diario Oficial No. 540704 del 31 de diciembre de 1991. Colombia.

Barros, J., y Rodríguez, R. (2004) Una nueva visión 
del cuadro de mando integral para el sector público. Revista iberoamericana de contabilidad de gestión, 1(4), 117-148.

Beltrán, O. (2005). Revisiones sistemáticas de la literatura. Revista Colombiana de Gastroenterología, 20(1), 60-69.

Calle, L. (2016). Metodologías para hacer la revisión de literatura de una investigación. Researhgate, 1(1), 1-8.

Callejas, A. (2019). Propuesta para fortalecer el sistema de gestión de la calidad organizacional en una entidad financiera del sector público colombiano. Signos, 11(1), 117-130

Camarasa, J. (2004). La calidad en la administración pública. Educar en el 2000, 8(2), 9-20.

Clement, G., y Rascoe, F. (2003). ETD Management and Publishing in the ProQuest System and the University Repository: A Comparative Analysis. Journal of Librarianship and Scholarly Communication, 1(4), 1-29.

Congreso de la República de Colombia. (1958). Ley 19 de 1958. Sobre reforma Administrativa. Publicada en El Diario Oficial No. 29835 del 9 de diciembre de 1958. Colombia.

Congreso de la República de Colombia. (1998). Ley 489 de 1998. Por la cual se dictan normas sobre la organización $\mathrm{y}$ funcionamiento de las entidades del orden nacional, se expiden disposiciones, principios y reglas generales para el ejercicio de las atribuciones en los numerales 15 y 16 del artículo 189 de la Constitución Política y se dictan otras disposiciones. Publicada en El Diario Oficial No. 43464 del 30 de diciembre de 1998. Colombia.

Congreso de la República de Colombia. (2003). Ley 872 de 2003. Por la cual se crea el sistema de gestión de la calidad en la Rama Ejecutiva del Poder Público y en otras entidades prestadoras de servicios. Publicada en El Diario Oficial No. 45418 del 30 de diciembre de 2003. Colombia.

Congreso de la República de Colombia. (2015). Ley 1753 de 1753. "Por la cual se expide el Plan Nacional de Desarrollo 2014 -2018 “Todos por un nuevo país". Publicada en El Diario Oficial No. 49538 del 9 de junio de 2015. Colombia.

Congreso de los Estados Unidos de Colombia. (1873). Ley 84 de 1873. Código Civil de los Estados Unidos de Colombia. Publicada en el Diario Oficial No. 2867 del 31 de mayo de 1873. Colombia.

De Nadae, J., Carvalho, M., y Rodrígues, D. (2019). Exploring the influence of environmental and social standars in integrated management system on economic performance of firms. Enginnering Economics, Journal of Manufacturing Technology Management, 30(52), 840-861.

Deming, W.E. (1982). Out of the Crisis. Cambridge: Massachusetts Institute of Technology, Center for Advanced Educational Services.

Departamento Administrativo de la Función Pública-DAFP. (2005). Decreto 1599 de 2005. Por el cual se adopta el Modelo Estándar de Control Interno para el Estado Colombiano. Publicado en El Diario Oficial No. 45920 del 26 de mayo de 2005. Bogotá: DAFP.

Departamento Administrativo de la Función Pública-DAFP. (2012). Decreto 2482 de 2012. Por el cual se establecen los lineamientos generales para la integración de la planeación y la gestión. Publicado en El Diario Oficial No. 47538 del 3 de diciembre de 2012. Bogotá: DAFP.

Departamento Administrativo de la Función 
Pública-DAFP. (2017). Decreto 1499 de 2017. Por medio del cual se modifica el Decreto 1083 de 2015, Decreto Único Reglamentario del Sector Función Pública, en lo relacionado con el Sistema de Gestión establecido en el artículo 133 de la Ley 1753 de 2015. Publicado en El Diario Oficial No. 50353 del 11 de septiembre de 2017. Bogotá: DAFP.

De Souza, J., y Macêdo, K. (2006). Cultura organizacional em organizações públicas no Brasil. RAP Rio de Janeiro, 40(1), 81-105.

Elkomy, S., y Cookson, G. (2018). Performance Management Strategy: Waiting Time in the English National Health Services. Public Organization Review, 1(1), 1-18.

Felcman, I., Blutman, G., y Parnes, S. (2002). Cultura organizacional en la Administración pública Argentina. Argentina: Ediciones Cooperativas.

Fernández, L. (2010). Bibliografía de William Edward Deming: El guru de la Calidad Total. (Tesis doctoral). Recuperado de http://genesismex.org/ACTIDOCE/CUR SOS/CHILE-CO-OT'10/GURUS/LORE NA-DEMING.pdf

Francesc, E., Duch Gavald, J., y Gisbert Cervera, M. (2014). Los aprendices digitales en la literatura científica: diseño y aplicación de una revisión sistemática entre 2001 y 2010. Pixel-Bit. Revista de medios y educación, (45).

García, I. (2007). La nueva gestión pública: evolución $\mathrm{y}$ tendencias. Instituto de Estudios Fiscales, 1(47), 37-64.

Gibson, J., Donnelly, J. H., Ivancevich, J., \& Konopaske, R. (2006). Organizaciones: comportamiento, estructura, procesos. México D.F.: McGraw-Hill.

Guirao-Goris, J. A., Olmedo Salas, A., y Ferrer Ferrandis, E. (2008). El artículo de revisión. Revista iberoamericana de enfermería comunitaria, 1(1), 1-25.

Gutiérrez Pulido, H., y Salazar, V. (2017). Control Estadístico de Calidad y Seis SIGMA. México: Editorial Mac Graw Hill.

Hood, C. (1991). A public management for all seasons? Public administration, 69(1), 3-19.

Hood, C., y Peters, G. (2004). The middle aging of new public management: into the age of paradox? Journal of public administration research and theory, 14(3), 267-282.

Hutyra, M. (2009). TQM Quality Management System as the basiss of quality assurance at VSB-Technical University of Ostrava. Polish Journal of Management Studies, 1(1), 121-128.

International Organization for Standardization (ISO) (2006). ISO 9001:2006: Managing for the Sustained Success of an Organization: A Quality Management Approach. International Organization for Standardization, ISO Central Secretariat, Geneva.

International Organization for Standardization (ISO) (2009). ISO 9001:2009: Managing for the Sustained Success of an Organization: A Quality Management Approach. International Organization for Standardization, ISO Central Secretariat, Geneva.

International Organization for Standardization (ISO) (2015). ISO 9001:2015: Managing for the Sustained Success of an Organization: A Quality Management Approach. International Organization for Standardization, ISO Central Secretariat, Geneva.

International Organization for Standardization (ISO) (2019). The ISO Survey of certifications to management system 
standards - Full results -2018. Recuperado en: https://isotc.iso.org/livelink/livelink?func $=11 \&$ objId $=18808772$ \&objAction $=$ brows e\&viewType $=1$

Joiner, B. L. (1995). Gerencia de la cuarta generación. México D.F.: McGraw-Hill.

Kaplan, R., y Norton, D. (2002). Cuadro de mando integral: the Balanced Scorecard. Bogotá: Editorial Planeta Colombiana S.A.

Khadra, H., Barqawi, B., y Alramahi, N. (2012). Governance using ISO 9001:2000 challenges and barriers: empirical study applied on the Jordanian private mobile companies. International Journal of Project Organisation and Management, 4(2), 171-202.

Lasrado, F. (2017). The excellence quest: a study of business excellence award-winning organizations in UAE. Benchmarking: An International Journal, 24(3), 716-734.

Legaz, A., Jové, C., Reverter, J., y Hernández, V. (2016). Producción en Web of Science y Scopus de profesores funcionarios con sexenio de las ciencias del deporte en España. Rev. Interam. Bibliot, 39(2), 55-68.

Lizarzaburu, E. (2016). La gestión de la calidad en Perú: un estudio de la norma ISO 9001, sus beneficios y los principales cambios en la versión 2015. Universidad $y$ Empresa, 18(30), 33-54.

Moyado, F. (octubre de 2002). Gestión pública y calidad: hacia la mejora continua y el rediseño de las instituciones del sector público. Ponencia presentada en el VII Congreso Internacional del CLAD sobre la Reforma del Estado y de la Administración Pública, Lisboa, Portugal.

Octavian, C., Stanciuc, A., y Viorel, B. (2013). Integrarea Managementului Total al
Calitãpii în administrapia publicã din România. Managementul calitãpii, 14(4), 28-35.

Osborne, S. (2006). The new public governance? Public Management Review, 8(3), 377-387.

Presidencia de la República. (1992). Decreto 2169 de 1992. Por el cual se reestructura el Departamento Administrativo del Servicio Civil. Publicada en El Diario Oficial No. 40704 del 31 de diciembre de 1992. Bogotá: Presidencia.

Presidencia de la República. (2004). Decreto 4110 de 2004. Por el cual se reglamenta la Ley 872 de 2003 y se adopta la Norma Técnica de Calidad en la Gestión Pública. Publicado en El Diario Oficial No. 45761 del 13 de diciembre de 2004. Bogotá: Presidencia.

Presidencia de la República. (2009). Decreto 4485 de 2009. Por medio de la cual se adopta la actualización de la Norma Técnica de Calidad en la Gestión Pública. Publicado en El Diario Oficial No. 47538 del 19 de noviembre de 2009. Bogotá: Presidencia.

Presidencia de la República. (2014). Decreto 943 de 2014. Por medio del cual se actualiza el Modelo Estándar de Control Interno (MECI). Publicado en El Diario Oficial No. 49158 del 21 de mayo de 2014. Bogotá: Presidencia.

Presidencia de la República. (2015). Decreto 1083 de 2015. Por medio del cual se expide el Decreto único Reglamentario del Sector de Función Pública. Publicado en El Diario Oficial No. 49523 del 26 de mayo de 2015. Bogotá: Presidencia.

Phusavat, K., Anussornnitisarn, P., Helo, P., y Dwight, R. (2009). Performance measurement: roles and challenges. Industrial Management \& Data Systems, 109(5), 646-664. 
Psychogios, A. (2010). A four-fold regional-specific approach to TQM: The case of South Eastern Europe. International Journal of Quality \& Reliability Management, 27(9), 1036-1053.

Ramírez, R., Meneses, J., y Florez, M. (2016). Una propuesta metodológica para la conducción de revisiones sistemáticas de la literatura en la investigación biomédica. CES Movimiento y Salud, 1(1), 61-73.

Rebelo, M., Santos, G., y Silva, R. (2015). Integration of management systems: towards a sustained success and development of organization. Journal

CreanerProduction, 1(1), 96-111.

Santiso, C. (1 de diciembre de 2016). La era de los gobiernos inteligentes. El País. Recuperado de:

https://elpais.com/internacional/2014/12/ 01/actualidad/1417469090_152983.html entes-carlos-santiso

Santos, M., y Álvarez, L. (2007). Gestión de la calidad total de acuerdos con el modelo EFQM: Evidencias sobre sus efectivos en el rendimiento empresarial. Universia Business Review, 1(3), 76-89.

Serna, H. (2008). Gerencia estratégica. Bogotá: 3R Editores.

Tanzi, V. (2000). El papel del Estado y la calidad del sector público. Cepal, 71(1), 7-22.

Tuomi, V., Ajmal, M., y Helo, P. (2013). Implementing TQM initiatives in public service organisations: case of academic libraries companies. International Journal of Productivity and Quality Management, 11(4), 393-411.

Valle, V. (2013). El papel del sector público en una economía moderna. eXtoikos, 1(9), 5-10.

Villegas, B. (2003). Rápida y pertinente búsqueda por internet mediante operadores booleanos. Unversitas Scientiarum, 8(1), 51-54.

Zeng, SX., Shi, JJ., y Lou, G. (2007). Towards effectiveness of integrated management system for enterprises. Enginnering Economics, 21(2), 177-179.

PODIUM No. 36, Diciembre 2019, pp. 35-54

(C) Universidad Espíritu Santo - UEES

ISSN: 1390-5473 e-ISSN: 2588-0969 